\title{
MPLP @ 5: More Access, Less Backlog?
}

Stephanie H. Crowe, Archivist, Charles Babbage Institute, University of Minnesota

Karen Spilman, Processing Coordinator, Archives and Special Collections, University of Minnesota

Author Posting. (c) Taylor \& Francis Group, LLC, 2010.

This is the author's version of the work. It is posted here by permission of Taylor \& Francis Group, LLC for personal use, not for redistribution.

The definitive version was published in Journal of Archival Organization, Volume 8 Issue 2, January 2010. doi:10.1080/15332748.2010.518079 (http://dx.doi.org/10.1080/15332748.2010.518079)

\author{
Abstract \\ Mark Greene and Dennis Meissner published their influential article "More Product, Less \\ Process: Revamping Traditional Archival Processing" five years ago. This study assesses the impact of \\ the methodologies expounded by Greene and Meissner on processing and reference in the archival \\ profession. A broad survey of American archivists conducted in the fall of 2009 is the basis for our \\ exploration of the extent to which the MPLP principles have thus far generally decreased collection \\ backlogs and increased researcher access to collections.

\section{Introduction}

In 2005, Mark Greene and Dennis Meissner published their landmark article "More Product, Less Process: Revamping Traditional Archival Processing." ${ }^{11}$ In this article, they decried traditional archival processing as hampering researcher access to materials due to the ever-increasing backlogs of unprocessed collections in archives everywhere. Instead, Greene and Meissner advocated an approach to processing that has become known as MPLP (or Greene-Meissner). Using this method, archivists would continue to fully process collections based on defined criteria, such as those with a high research or monetary value, at a detailed level. Most collections, however, would be physically processed at a much reduced level, one that matches the level of description. Such an approach, according to the authors, would reduce the backlog created by newly acquired and previously accessioned but unprocessed collections, thus providing access to more materials. 
While this approach was not necessarily a radical shift to archivists who had already been practicing certain aspects of this philosophy for a number of years, Greene and Meissner were the first to articulate a different approach to collections care and management based on an exhaustive review of the literature and of available data. ${ }^{2}$ Greene and Meissner's article has created a great deal of discussion in the archival community. Since the profession has now reached the five-year anniversary of MPLP, and given that the method has now been tacitly endorsed by the Society of American Archivists (SAA) and other archival organizations through instructional workshops, we felt that it would be an opportune time to survey the archival profession to determine how many archives have instituted a form of MPLP and what effect it has had on collection backlogs, reference practices, and researcher access.

\section{Literature Review}

Much of the post-Greene-Meissner literature highlights case studies of implementation in particular institutions. For instance, in the Fall/Winter 2006 issue of American Archivist, Christine Weideman discusses the application of MPLP, and minimal processing standards in general, to collections at Yale University. Stating that "accessioning as processing is now the goal," Weideman details the process in her department of using MPLP standards to prevent incoming collections from being added to their backlog. ${ }^{3}$ As in Weideman's article, the vast majority of publications and conference presentations about case studies have shown positive results on reducing processing backlogs using MPLP. In a 2006 article, Donna McCrea details a new approach at the University of Montana at Missoula to collections management that she states was "largely inspired by" Greene's and Meissner's recommendations. This methodology helped her repository to decrease both its backlog and its processing time per cubic foot of material. ${ }^{4}$ Similarly, a number of conference presentations have highlighted the benefits of MPLP practices at specific repositories. Various conference sessions have covered the application of MPLP to visual materials and corporate records, as well as broadly across institutions, while appropriately addressing privacy and security concerns. ${ }^{5}$ 
A pilot fast processing project carried out at the University of Minnesota in the spring of 2008 experimented with using centralized student staff, with a small amount of professional supervision, to minimally process organizational records and personal papers from a variety of archives and special collections repositories. The project had the two-pronged goal of decreasing collection backlogs and increasing storage space, and resulted in a processing rate of one hour per cubic foot. ${ }^{6}$

Although most case studies focus on an institution's implementation of the "minimal processing" aspect of Greene and Meissner's recommendations, a different perspective is contained in Sabre and Hamburger, "A Case for Item-Level Indexing: The Kenneth Burke Papers at the Pennsylvania State University." Sabre and Hamburger write that "despite Mark Greene and Dennis Meissner's recent recommendations to process lightly, valid reasons remain for continuing traditional practices for many literary correspondence collections." ${ }^{7}$

Though sparser than the case studies, there is also a body of literature analyzing MPLP for various purposes beyond a single institution. One recent focus in the profession has been on the effects of MPLP processing standards on reference work. At the 2007 annual SAA conference, one session was devoted to the "challenges and opportunities" of MPLP on reference activities, stating in part that reference archivists should be more involved in determining the application of MPLP to collections. ${ }^{8}$ Additionally, in 2008, the SAA Reference, Access, and Outreach Section published an MPLP Task Force Report summarizing its members' thoughts on MPLP and its impact on reference. The report concluded that respondents, overall, were "supportive of MPLP concepts," though the issues that were raised included an inability to discover materials for researchers, a difficulty with security and physical control of materials when used by researchers, minimal processing presenting a barrier to new researchers, and an increase in the amount of time necessary for staff to assist researchers. ${ }^{9}$

MPLP's effect on public services has also drawn attention from the Rare Books and Manuscripts Section of the Association of College and Research Libraries: a panel at its 2009 preconference focused 
on the influence of MPLP on users and reference archivists. In particular, Shannon Bowen's extensive collection of data on the effects of MPLP processing on both researchers and public service archivists, presented at this panel session, provides useful insight into the perspectives of reference staff and users when dealing with more minimally processed collections. ${ }^{10}$ Additionally, Tiah Edmundson-Morton performed a case study testing users' responses to minimally processed collections, finding that users cared more about having a context for their materials than an item-level inventory. ${ }^{11}$ MPLP has additionally been discussed in terms of its efficacy for processing electronic records. Greg Johnson wrote in 2007 that MPLP standards can and should be adapted for electronic formats of materials. ${ }^{12}$

It has been clear that since the publication of the Greene-Meissner article, archivists have held widely differing opinions about MPLP practices. Jeffrey Suchanek, in an MPLP session at the SAA annual meeting in 2009, provided some reasons for believing that MPLP has been too indiscriminately applied by many different institutions, stating that it will have a negative effect on donor relations, prevention of theft, and possibly even collection backlogs. ${ }^{13}$ Furthermore, recent discussion on the Archives and Archivists listserv in August 2009, right around the time of our survey, suggested that some archivists disagree with the fundamental principles outlined in the article - primarily, the idea that collections will be useful and accessible to researchers when processed at a very basic level. For example, one archivist wrote, "...My researchers and users are incredibly given to specific terms and quests, possibly as a byproduct of Google and the internet. It is not clear to me how MPLP can ignore the basic need of access and description without loss of credibility." ${ }^{14}$

At the same time, many archivists have begun to formulate their own processing frameworks that have been, to a greater or lesser extent, based on Greene and Meissner's recommendations. For example, Dan Santamaria wrote, "Often missed in the discussions about MPLP is that the article's most important recommendation is to provide a baseline level of access to ALL collections in a repository before moving on to more detailed processing. The impact of "minimally" processing one collection is 
negligible. It's very easy as a processing archivist to focus on individual collections, but we need to think about the big picture of providing access to our entire holdings."15 Additionally, Robert Cox has outlined the "maximal processing" approach to collections management used at the University of MassachusettsAmherst, which he describes as providing comprehensive access to the repository's entire scope of collections while individual collections are queued for fuller processing based on a number of different factors. ${ }^{1617}$

In this study, we attempt to provide a data-driven context for archivists' experiences with MPLP at their individual institutions, their perception of its effects on processing and reference services in their repositories, and their own, personal opinions of how MPLP has affected, and will continue to affect, the archival profession.

\section{Methodology}

To begin to determine the effects of MPLP on the archival profession after its first five years, we decided to administer an electronic survey to the archives community. We created the survey in an online program called Survey Monkey (www.surveymonkey.com), to which the University of Minnesota holds a subscription. On August 3, 2009, we submitted the survey to the Archives and Archivists listserv, which is the foremost listserv for archivists nationally, managed by the Society for American Archivists.

In the survey (results available in Appendix 1), we asked an initial set of screening questions designed to funnel respondents to specific sections. If respondents were unfamiliar with MPLP as a concept, or if they did not have a supervisory or decision-making role in their repositories, they were immediately sent to a thank-you page. Remaining respondents were funneled to one of three sections based on their self-identified role: processing, reference, or both. One final screening question was asked at the beginning of each of the three sections regarding whether MPLP had been implemented at the respondent's repository. We deliberately left out a definition of MPLP so that respondents could determine for themselves if they had implemented it. If they had not used MPLP, respondents were 
asked only why they had not, and then were sent to the end. All other respondents were asked a series of questions based on their role. Based on the answers we received in each of the three sections of the survey, we hoped to learn about the effects of MPLP on both processing and reference services in the archival profession: specifically, whether its implementation has in fact resulted in a decrease in backlogs, as well as whether it has improved researchers' ability to access collections.

\section{Results and Analysis}

\section{Demographic Information}

The survey was open from August 3, 2009 through August 28, 2009, at which point we closed it to further responses. We received 156 responses during that period, of which only two respondents were unfamiliar with the concepts presented in the Greene-Meissner article. Although we recognize that the survey was unscientific and that the number of respondents was small compared to the number of archivists on the A\&A listserv, we believe that we received enough data to reflect the practices and beliefs of the archival community.

Of the respondents who were familiar with MPLP, 40 listed themselves as responsible for processing and collections management only, while 99 claimed responsibility in both processing and reference. We received no responses from archivists responsible for only reference services, possibly a result of MPLP being a topic generally of more interest to processing archivists.

Along with general job classification, we asked respondents to identify themselves based on the type of institutions in which they worked, the number of years' experience they had in the profession, the number of paid FTEs working in their repositories, the number of volunteers and student staff working in their repositories, and the types of materials held in their repositories. The majority of respondents worked in academic libraries (about 57\%), with government archivists as the only other category over 10\%. [Table 1a] Respondents were fairly evenly divided in terms of years in the archives profession, ranging from $13 \%$ with $0-2$ years of experience to $25 \%$ with $5-10$ years. [Table 1 b] Most 
archivists who responded worked in fairly small repositories, with $44 \%$ overall in repositories with $2-5$ full-time staff members and 53\% with 1-5 volunteers and/or student staff. [Tables $1 \mathbf{c}$ and $1 \mathrm{~d}$ ] We broke down respondents into these different categories because we were curious if there would be differences in MPLP implementation and effects among archivists at various types of institutions, with different lengths of time in the profession, and at different size repositories. However, with the exception of the archivists at academic institutions, no sub-category in type of institution, amount of professional experience, or repository size was large enough to provide a useful sample for the purposes of analysis. We compared academic archivists' responses to the responses of the whole group, and it appeared that the subgroup of archivists at academic institutions was fairly representative of the entire group of respondents.

Altogether, of the 139 respondents who had knowledge of MPLP and had some sort of decisionmaking responsibility in processing or reference services, 79\% (110 respondents) had implemented some aspect of MPLP. It is possible, though not necessarily the case, that this number is higher than in the archival community in general, due to potentially a greater interest in an MPLP survey among archivists who are already implementing it in some form.

Processing

All respondents to the survey indicated they had some processing responsibility, with 40 being primarily responsible for processing and 99 having responsibility in both processing and reference services. There are slight, but perhaps not statistically significant, differences in implementation of MPLP between archivists exclusively responsible for processing and those that handle both processing and reference: $85 \%$ of respondents who self-identified as processors only have implemented MPLP, while just under $77 \%$ of those that handle both processing and reference have done so.

Overall, $78 \%$ of respondents have applied MPLP to their incoming collections. When this number is broken down by responsibility (processing vs. processing and reference services), $64 \%$ of 
processors have applied MPLP to their incoming collections while $84 \%$ of those responsible for processing and reference services have done so. This disparity between the two groups was unexpected so we investigated further to see if the type of archival repository made a difference. When looking specifically at academic Institutions, the only institutional type with a large enough sample size, this disparity was repeated. $68 \%$ of those with only processing responsibilities have implemented MPLP on incoming collections, while $91 \%$ of those with responsibility in processing and reference have applied MPLP to their incoming collections. One possible explanation for this discrepancy is that archivists with dual/multiple responsibilities have less time to devote to processing, making MPLP more appealing.

Applying MPLP to collection backlogs is comparable for both groups, with $81 \%$ implementation for those with responsibility for processing and $79 \%$ for both processing and reference services. Since Greene and Meissner's article focused on implementing minimal processing procedures to decrease collection backlogs, a high favorable response rate to this question was not surprising.

When broken down by type of material, MPLP is most often applied to personal papers, corporate/business records, and institutional records. When looking at the numbers by responsibility, archivists who self-identified as processors only applied MPLP most often to personal papers (72\%), corporate/business records (68\%), and institutional records (56\%). [Table 2a] Archivists responsible for both processing and reference applied MPLP most often to institutional records (66.2\%), personal papers (61.8\%), and corporate/business records (48.5\%). [Table $\mathbf{2 b}$ ] Regardless of responsibility, about one-third have used MPLP on photograph or slide collections, and approximately one-quarter have implemented on published materials. In much lesser numbers, archivists have also implemented MPLP on maps, architectural drawings, government records, artifacts, artwork, moving images, and borndigital materials. ${ }^{18}$

Common applications of MPLP to both backlog and incoming collections include creating a basic catalog or EAD record for each collection, limiting or eliminating refoldering and removal of metal 
fasteners, describing collections at the collection level or the box level rather than folder or item level, and approaching collections on a tiered basis to determine how fully they should be processed. For example:

- "Records are created and items processed minimally and shelved unless they need immediate attention due to damage, age or other factors."

- "All collections receive a box-level inventory. In some cases, finding aids are created off of this. These collections are accessible to patrons."

- “Initial 'adequate' processing done immediately upon acquisition. More intensive processing done on as-needed basis."

- "Doing box level inventory to have basic intellectual/physical control over items."

- "We have created a partnership with government agencies transferring records to the archives to provide basic box and file level data."

- "We are no longer going through the collection removing all staples and paperclips or even opening up individual files. I've also accepted that collections don't have to be processed before granting access."

- "Most incoming collections are arranged and described at the collection level as soon as possible after receipt. More detailed processing is only applied to specific types of collections and all decisions to process in detail must be weighed against the value for researchers versus other collections waiting for processing."

- "We inventory and rehouse collections as they come in and create a finding aid, but in most cases little is done to them beyond that (although of course it depends on the nature of the collection, its preservation issues, etc.)."

- "Nothing is arranged within folders, although items without folders are placed in them, making some effort to impose some order to collections which are totally unprocessed. In most cases, however, we 
simply survey the contents in order to gather enough knowledge to create a catalog record for uploading to our OPAC."

- "MPLP is the default way we process collections unless we know for a fact that the collection will receive high use ... in the latter case, we process more thoroughly."

Our main concern in the area of collection processing was to determine whether the application of MPLP has helped to decrease repositories' backlogs. Of the 75 archivists who responded to that question, 49 (65\%) indicated that they believed their backlogs had decreased slightly or significantly since their implementation of MPLP. Of those, nearly $80 \%$ said that their decrease in backlog was a direct result of MPLP, while just one individual said positively that it was not (nine were unsure). [Tables 3a and 3b] Such a result is encouraging for the usefulness of MPLP procedures.

\section{Reference}

However, a reduction in backlog is not helpful unless it also increases access to collections for researchers. Although no respondents self-identified as being responsible for only reference, we were able to gather some data about MPLP's effect on public services and researchers based on the responses given by those in charge of both processing and public services in their repositories. While $37 \%$ of respondents ( 25 of 67 ) responded that their ability to assist researchers with inquiries has remained the same since their repository's adoption of MPLP, an additional 39 respondents (58\%) replied that their ability to assist researchers has increased slightly or significantly. Only three respondents believed that such ability has decreased slightly, and none thought it had decreased significantly. [Table 4] Of the respondents who answered positively, $82 \%$ believed that an increase in ability to help researchers was a direct result of their repository's MPLP implementation.

We received similar results for researchers' ability to access collections: nearly $29 \%$ said that it remained the same since their repository's MPLP implementation, while almost $70 \%$ said that it increased slightly or significantly. A smaller majority believed that increased access was a direct result 
of MPLP, at $63 \%$. Altogether, since increased access to collections is the ultimate goal of MPLP, the results of this survey were quite encouraging as to its benefits.

Non-Implementers and Further Issues

Respondents who have not implemented MPLP at their repository were asked to explain why. Eleven, almost one-third of respondents, noted they require a higher level of processing to access their collections. Reasons as to why vary, including collections stored off-site requiring detailed finding aids to retrieve appropriate boxes, concern over allowing access to private data, and inability to find candidates for digitization. Four respondents said they are planning to implement MPLP soon, and three others noted they had already implemented minimal processing before the publication of MPLP. Two noted that MPLP is not appropriate for their collections. Only one person noted that MPLP is the "wrong solution" to processing backlogs, while another said it is a "drastic" approach to the problem. The majority of those that have not implemented MPLP at their repositories have not done so either because they feel it is not appropriate for their collections or does not meet their needs; only a couple of respondents were strongly opposed to MPLP.

One result we did not anticipate was the number of respondents who stated that they had already practiced some form of minimal processing before the Greene-Meissner publication and that MPLP, in effect, should not be receiving all the credit for minimal-type processing. Indeed, Greene and Meissner mentioned as much in their article, stating that "the recommendations that follow simply give voice to the small but growing number of archivists who have quietly abandoned traditional approaches to processing."19 In retrospect, however, perhaps it would have been helpful for us to clearly state our understanding of this fact at the beginning of the survey, rather than assuming that respondents would answer based on their practices whether or not they had begun such practices with the publication of the Greene-Meissner article. 
Finally, it was clear from our results that some archivists have a fundamental misunderstanding of the principles behind MPLP. These archivists view it as a doctrine requiring everything to be processed at a minimal level. Rather, Greene and Meissner advocate that minimal-level processing be used broadly, leaving the option for some collections, or some series, to be processed more fully. ${ }^{20}$ Future of MPLP

At the end of the survey, we asked all individuals, whether or not they had implemented MPLP, to respond to an optional question on what they viewed as the future effects of MPLP. We received 100 responses that fell into several main groups:

a) The influence of MPLP and minimal processing will result in a loss of professional status among archivists: "I think it's a good thing to get brief descriptions out there but at some point we're going to lose our professional status if we don't know what's in the collections."

b) MPLP will ultimately hurt researcher access to collections: "I think it will make reference work more difficult and place the burden on the researcher."

c) MPLP will ultimately help researcher access to collections: there will be "considerable increase in researchers' ability to locate and use collections."

d) MPLP will help open more collections more quickly: "Many more collections will be made accessible, though this depends on online collection descriptions and finding aids being made available as well. MPLP needs that second component of discovery tools and information for it to truly increase collection visibility and access."

e) MPLP could cause issues with privacy, security, and preservation: "I think it will provide access to huge amounts of backlog materials, but I wonder what the short-term repercussions will be for security/privacy and in the long-term how it will affect the longevity and preservation of materials since we don't all have repositories or storage with perfect environmental conditions." 
f) MPLP will fade out in the next decade, with an increased focus on more detailed processing and description due to digitization: "More collections will have a descriptive handle, but we'll know less about the content of the collections and therefore the ways in which they may be useful to researchers. I predict that within the next ten years, the pendulum will swing back and there will be another overhaul of archival processing focused on detailed description, especially at the item level related to mass digitization."

Other issues mentioned included effects on donor relations, decrease in supply costs, decreased ability to digitize and describe digital collections, effects on collection management strategies, effects on accessioning practices, and changes in descriptive practices.

It is interesting to note that while the quantitative results of the survey were overwhelmingly positive as to the effects of MPLP, a surprising percentage of the freeform comments on the future of MPLP were ambivalent or negative. Although we have no way of determining why this is the case, it is possible that some of those who have implemented MPLP are actually undecided as to its future benefits for the profession.

\section{Conclusions/Further Thoughts and Future Possible Studies}

Based on the results from this study, it appears that MPLP has been widely accepted in the archival community. The responses indicated a positive effect of MPLP procedures on processing backlogs, researcher access, and the ability of reference staff to more easily assist researchers in their quests. Overall, $78 \%$ of respondents had applied MPLP to incoming collections, and $79 \%$ to collection backlogs. $65 \%$ of these individuals indicated that their backlogs had decreased since applying MPLP practices. $58 \%$ noted an increased ability to assist researchers with their queries, while $70 \%$ believed that their researchers had increased access to the repository's collections.

However, our research is only a starting point. There are a number of areas we identified for future research on the effects of MPLP and on processing practices as a whole. The number of 
responses to this survey did not allow for detailed analysis comparing MPLP's effects at different types of archival repositories (e.g. academic versus government), among archivists with different levels of experience, or among different sizes of institutions. If a larger sample size could be accumulated, perhaps through broader submission to targeted archives working groups and roundtables, such a comparative analysis would be useful. Further, a comparison to international archivists would be interesting, as our survey was geared towards Americans. What effect, if any, has MPLP had on archivists beyond the United States?

A further study could also be geared towards the effect of MPLP on descriptive practices, including MARC catalog records and finding aids, including those encoded in EAD. Have archivists accepted Greene and Meissner's recommendation that description of a collection should match the level of arrangement ${ }^{21}$ If so, have these descriptive practices affected researchers' ability to find appropriate materials? User studies such as that conducted by Shannon Bowen would be an area worth pursuing further. Further research into users' preferences regarding the level of processing detail would additionally be useful. ${ }^{22}$

Greene and Meissner note in their conclusion that the only feasible way to reduce processing backlogs is to " ... change the way we process so that we can, with our existing resources, roughly triple the speed with which we process. This is exactly what we propose, although many archivists will probably find it hard to believe that we are serious." ${ }^{23}$ This study shows that five years after the Greene-Meissner article, many archivists in fact have come to take their proposition extremely seriously.

\section{Appendix 1: Raw Results}

\footnotetext{
${ }^{1}$ Mark A. Greene and Dennis Meissner, “More Product, Less Process: Revamping Traditional Archival Processing," The American Archivist 68 (Fall/Winter 2005).
} 
${ }^{2}$ See, for example, Maxine B. Clapp and Clodaugh Neiderheiser, "Box Label Inventory," The American Archivist 29, no. 3 (July 1966). Clapp and Neiderheiser, archivists at the University of Minnesota, described a method they called "preliminary processing," in which they did not rearrange or refolder materials, but rather prepared a "detailed" box inventory which they attached to the front of boxes and added to the card catalog. The authors emphasized that this method should not take the place of detailed processing but rather provided a method whereby "rapid retrieval" of collections could occur.

${ }^{3}$ Christine Weideman, "Accessioning as Processing," The American Archivist 69 (Fall/Winter 2006): 276.

${ }^{4}$ Donna E. McCrea, "Getting More for Less: Testing a New Processing Model at the University of Montana," The American Archivist 69 (Fall/Winter 2006).

${ }^{5}$ For example, “'More Product, Less Process': New Processing Guidelines to Reduce Backlogs" (Society of American Archivists annual meeting, New Orleans, Louisiana, August 19, 2005); “'More Product' in the Image Archives: Applying Minimal Processing Guidelines to Visual Materials Collections" (SAA annual meeting, Washington, DC, August 3, 2006); "Adopting Triage or Accession-Level Processing as a Standard for Certain Types of Corporate Records: A Look at Royal Bank of Canada's Example” (SAA annual meeting, Chicago, IL, August 30, 2007); “MP-LP Comes Home to Roost: Applying the Greene-Meissner Recommendations Broadly Across an Institution" (SAA annual meeting, Chicago, IL, August 30, 2007); and "More Product, Less Privacy? Applying Minimal Processing with an Awareness of Sensitive, Confidential, or Restricted Collection Materials" (SAA annual meeting, Chicago, IL, August 31, 2007).

${ }^{6}$ We were both instrumental in the creation, implementation, and management of this project, which contributed to our interest in MPLP and its applications beyond our own institution. Although the processing rate was much faster than quoted even in Greene-Meissner (who mention a rate of four hours per cubic foot), this was in part a result of deliberately selecting collections that contained a large amount of known duplicative and problematic material that could quickly be identified and weeded.

7 Jeannette Mercer Sabre and Susan Hamburger, "A Case for Item-Level Indexing: The Kenneth Burke Papers and The Pennsylvania State University," Journal of Archival Organization 6 (August 2008): 25. The first part of this statement is somewhat misleading and demonstrates a common misconception among archivists regarding Greene and Meissner's proposal, as Greene and Meissner in their original article acknowledge the fact that some circumstances and some collections continue to require a more detailed level of processing.

8 "Reference Service and Minimal Processing: Challenges and Opportunities" (SAA annual meeting, Chicago, IL, August 30, 2007).

${ }^{9}$ Society of American Archivists Reference, Access, and Outreach Section, MPLP Task Force Report, http://www.archivists.org/saagroups/rao/MPLPTF survey report.pdf (accessed January 15, 2010).

${ }^{10}$ Shannon Bowen, "Unhidden Collections and the People Who Love Them: What We Know About Use and Users on Both Sides of the Reference Desk" (RBMS annual preconference, Charlottesville, VA, June 17-20, 2009), http://rbms.info/conferences/preconfdocs/2009/SeminarG bowen.pdf (accessed February 15, 2010). From seminar panel "Public Services and 'Un-Hidden' Collections: What We Know and What We Need to Know."

${ }^{11}$ Tiah Edmundson-Morton, "SAA 2007 Session \#307," Archival Musings blog, posted September 4, 2007, http://www.temarchivalmusings.blogspot.com/2007/09/saa-2007-session-307.html (accessed January 21, 2010).

${ }^{12}$ Gregory P. Johnson, "Quality or Quantity: Can Archivists Apply Minimal Processing to Electronic Records?" (Master's paper, University of North Carolina, April 2007).

http://etd.ils.unc.edu/dspace/bitstream/1901/413/1/gregjohnson.pdf (accessed February 15, 2010).

${ }^{13}$ Jeffrey S. Suchanek, "More Product, Less Process: One Size Does Not Fit All," (SAA annual meeting, Austin, TX, August 15, 2009). http://www.archivists.org/conference/austin2009/docs/Session501-Suchanek.doc (accessed February 15, 2010).

14 “Processing decisions/MPLP” thread, Archives and Archivists listserv, posted August 19, 2009, http://forums.archivists.org/read/messages?id=33336\#33336 (accessed January 26, 2010).

${ }^{15}$ Dan Santamaria, "Guest blogger: Dan Santamaria shares some thoughts on the recent MPLP discussions," ArchivesNext blog, posted August 21, 2009, http://www.archivesnext.com/?p=332\%29 (accessed January 21, 2010). Santamaria recognizes, unlike some archivists involved in this debate, that MPLP does not equate with minimal-level processing across the board. 
${ }^{16}$ Robert S. Cox, "Maximal Processing, or, Archivist on a Pale Horse" (precirculated paper, New England Archivists Spring 2010 Meeting, March 19-20, 2010),

http://www.newenglandarchivists.org/meetings/maximal processing cox.pdf (accessed February 16, 2010 ): 15.

${ }^{17}$ Since submitting this article for publication, we have become aware of two more recent pieces relating to MPLP as a processing theory. Though too late for incorporation into our analysis, we want to acknowledge these articles as further readings on this topic. See Carl Van Ness, "Much Ado about Paper Clips: 'More Product, Less Process' and the Modern Manuscript Repository," The American Archivist 73 (Spring/Summer 2010): 129-145; and Mark A. Greene, "MPLP: It's Not Just for Processing Anymore," The American Archivist 73 (Spring/Summer 2010): 175-203.

${ }^{18}$ The exception, of course, is government archivists, who have implemented MPLP on government records at a much higher rate than their peers at other types of repositories.

${ }^{19}$ Greene and Meissner, p. 240.

${ }^{20}$ Greene and Meissner, p. 233.

${ }^{21}$ Greene and Meissner, 246.

${ }^{22}$ Tiah Edmundson-Morton's research cited above is a useful starting point for this type of study.

${ }^{23}$ Greene and Meissner, 254. 